\title{
The Impact of Political, Legal and Economic Institutions on Family Takaful/ Life Insurance Consumption in OIC Countries
}

Mohammad Ghaith Mahaini*, Kamaruzaman Noordin, Mohammad Taqiuddin Mohamad*

Department of Shariah and Economics, Academy of Islamic Studies, University of Malaya, 50603 Lembah Pantai Kuala Lumpur

*Corresponding author: m.taqiuddin@um.edu.my

\section{Article history}

Received: 2019-04-25 Received in revised form: 2019-08-19 Accepted: 2019-08-20 Published online: 2019-10-31

\begin{abstract}
This study aims at testing the impact of political, legal and economic institutions on life insurance/ family takaful consumption in OIC countries. Using a panel data covering 33 OIC countries for the years from 1990 till 2016, fixed effects and random effects models have been utilised. The empirical results suggest that for political institutions, more government effectiveness promotes consumption of life insurance in OIC countries. Additionally, the more unstable the country is, the more life insurance/family takaful is purchased perhaps as an attempt of individuals to mitigate the increased level of risks. Similarly, economic institutions, measured by both investment freedom and financial freedom, have a positive impact on life insurance consumption in OIC countries. However, results show that trade freedom index has a negative impact. Further, legal institutions do not seem to have any significant impact on life insurance consumption in OIC countries.
\end{abstract}

Keywords: Institutions, insurance, takaful, OIC. 


\subsection{INTRODUCTION}

Protection against perils through insurance/takaful is considered one of the three pillars of the financial system. The global insurance gross written premium amounted to USD 4.8 trillion in 2017 (Crawford, Kumar, \& Russignan, 2018). Still, OIC countries tend to have inferior levels of life insurance penetration rates when compared to developing non-OIC countries. World Bank data shows that the 27 years average of life insurance penetration rate is as low as approximately 0.31 percent of total GDP in OIC countries. This ratio is very small compared to non-OIC developed and developing countries with life penetration rates of 2.81 and 1.30 percent, respectively. Scholars claim that being a Muslim majority country hinders life insurance growth based on religious beliefs. However, life insurance penetration growth is stagnant even after years of introducing family takaful products to many major OIC economies.

Literature on the determinants of life insurance consumption is abundant (Alhassan \& Biekpe, 2016; Beck \& Webb, 2003; Beenstock et al., 1986, 1988; Browne et al., 2000; Browne \& Kim, 1993; Elango \& Jones, 2011; Esho et al., 2004; Hammond, Houston, \& Melander, 1967; Kjosevski, 2012; Mantis \& Farmer, 1968; H. Park et al., 2002; S. C. Park \& Lemaire, 2012; Sen \& Madheswaran, 2013; Ward \& Zurbruegg, 2002). Several studies categorised life insurance determinants into three main categories, namely economic, social-demographic and institutional determinants (Alhassan \& Biekpe, 2016; Beck \& Webb, 2003; Elango \& Jones, 2011; Kjosevski, 2012; Sen \& Madheswaran, 2013; Ward \& Zurbruegg, 2002).

North (1990) suggested that institutions are constraints devised by humans that structure political, economic and social interaction. He opined that institutions consist of both formal and informal constraints that include taboos, customs and traditions as well as constitutions, laws and property rights. Since the work of North (1990) and the development of the new institutional theory, more attention has been given to the role of the quality of institutions on the fields of economic growth (Brunetti, Kisunko, \& Weder, 1997; Butkiewicz \& Yanikkaya, 2006; Chong \& Calderón, 2000; Klein et al., 2005; Knack \& Keefer, 1995; North, 1990; Redek \& Sušjan, 2005; Rodrik, 2000; Siddiqui \& Ahmed, 2013), financial development (Bhattacharyya \& Hodler, 2014; Demetriades \& Law, 2006; Law \& Azman-Saini, 2008; Outreville, 1999; Roe \& Siegel, 2011), and Trade (Anderson, 2001; Anderson \& Marcouiller, 2002; Dollar \& Kraay, 2003; P. G. Méon \& Sekkat, 2008; P. Méon \& Sekkat, 2002; Grigorian \& Martinez, 2001). Further, Kunčič (2014) suggested that there are heterogeneous groups of institutions that can be categorised as: Political, legal and economic institutions. Institutions aim to lower risks through reducing uncertainty and transaction cost. since insurance/ takaful services are tools for manging risks, it is expected that institutions have an impact on insurance/takaful consumption. This study is conducted to test the impact of the three categories on life insurance/ family takaful consumption in OIC countries for the period between 1990 till 2016. Despite agreeing that insurance and takaful are different in their contractual structure and business model, it can be argued that both are designed to serve similar purposes. Therefore, this paper uses insurance premiums to proxy for both life insurance and family takaful -whenever takaful is available in the country-combined. This is also done due to the lack of available macroeconomic indicators of takaful data across OIC countries. The paper is organized as follows. Section 2 outlines literature about life insurance determinants. Section 3 presents the data and methodology. Section 4 discusses the empirical results and we conclude with Section 5 . 


\subsection{LITERATURE REVIEW}

The theoretical underpinnings for the demand of life insurance were initiated by Yaari (1965) who introduced a model for life insurance demand. The author opined that the uncertain lifetime and time of death lead individuals to demand life insurance to improve life utility. Hakansson (1969) added wealth, income, price of insurance and interest rates to the model as other variables that may impact consumption of insurance. Fischer (1973) opined that individuals who live off wealth proceeds are unlikely to purchase life insurance while individuals who receive labour income are likely to do so during the early stages of their lives. These findings were in congruent with the general findings of Mossin (1968); Pratt (1964); and Smith (1968).

A quick review of the literature shows that the significant economic determinants of insurance consumption are, namely level of income (Alhassan \& Biekpe, 2016; Beck \& Webb, 2003; Beenstock et al., 1986, 1988; Browne et al., 2000; Browne \& Kim, 1993; Elango \& Jones, 2011; Esho et al., 2004; Hammond et al., 1967; Kjosevski, 2012; Mantis \& Farmer, 1968; H. Park et al., 2002; S. C. Park \& Lemaire, 2012; Sen \& Madheswaran, 2013; Trinh et al., 2016; Ward \& Zurbruegg, 2002), real interest rates (Beck \& Webb, 2003; Beenstock et al., 1986, 1988; Elango \& Jones, 2011; Kjosevski, 2012; Sen \& Madheswaran, 2013), inflation (Alhassan \& Biekpe, 2016; Beck \& Webb, 2003; Browne \& Kim, 1993; Elango \& Jones, 2011; Kjosevski, 2012; Mantis \& Farmer, 1968; Sen \& Madheswaran, 2013; Ward \& Zurbruegg, 2002) and financial development (Alhassan \& Biekpe, 2016; Beck \& Webb, 2003; Kjosevski, 2012; Sen \& Madheswaran, 2013; Trinh et al., 2016; Ward \& Zurbruegg, 2002). The social-demographic determinants are, namely risk aversion measured by education (Alhassan \& Biekpe, 2016; Beck \& Webb, 2003; Browne et al., 2000; Elango \& Jones, 2011; Esho et al., 2004; Kjosevski, 2012; S. C. Park \& Lemaire, 2012; Sen \& Madheswaran, 2013; Trinh et al., 2016; Ward \& Zurbruegg, 2002) and life expectancy (Alhassan \& Biekpe, 2016; Beck \& Webb, 2003; Beenstock et al., 1986; Elango \& Jones, 2011; Sen \& Madheswaran, 2013; Ward \& Zurbruegg, 2002).

The focus on the role of institutions on life insurance consumption has started only around the beginning of the new millennium. The majority of papers looking into determinants of insurance consumption that took institutions into account merely focused on one or two indicators only. Mostly, studies focused on the effect of legal system, e.g. (Browne et al., 2000; Esho et al., 2004; S. C. Park \& Lemaire, 2012; Trinh et al., 2016). Another common approach is to take the average of different institutional variables and create an institutional index, e.g. (Alhassan \& Biekpe, 2016). This method does not allow to capture the roles of different types of institutions i.e. the legal, political and economic institutions although it does fuse all of them into one indicator. Only very few papers looked into the three different types of institutions (Elango \& Jones, 2011; Ward \& Zurbruegg, 2002). The former looked into factors driving insurance demand in emerging markets during the years 1998 till 2008 whilst the latter looked into determinants of life insurance consumption in Asia compared to developed/OECD countries for the period between 1987 and 1998.

Additionally, the focus on life insurance determinants in OIC countries seems to be limited to adding a control variable for being a Muslim majority country which may have a significant negative impact on insurance consumption (Beck \& Webb, 2003; S. C. Park \& Lemaire, 2012; Ward \& Zurbruegg, 2002). Finally, Chang \& Lee (2011) indicated that political circumstances have a positive impact on life insurance especially in low income countries.

These studies along with similar conclusions (Esho et al., 2004; S. C. Park \& Lemaire, 2012) suggest that life insurance consumption, as well as the role of institutions are heterogeneous in 
nature across the globe. Hence, the determinants of insurance consumption and the role institutions for OIC countries need to be studied in particular. To the best of our knowledge, no paper has looked into how institutions affect insurance development in OIC countries.

\subsection{METHODOLOGY}

\subsection{Variables And Data}

Based on the above review, it can be suggested that theoretical models proposed by Yaari (1965), Hakansson (1969) and Lewis (1989) for life insurance consumption have been the starting models of empirical studies. As mentioned earlier, empirical studies suggest that insurance consumption is a function of three main time-variant factors. These factors are economic, socialdemographic and institutional factors. Hence, the empirical model for this study can be denoted as follows:

$$
I_{i, t}=\alpha_{i}+\beta_{t}+K_{t} C_{i t}+K_{t} D_{i t}+K_{t} Q_{i t}+\epsilon_{i t}
$$

Where: $\alpha_{\mathrm{i}}$ is a dummy variable to control for country-specific factors that are constant over time (e.g., geographical factors, legal system factor, religion); $\beta_{t}$ is a dummy variable to control for time-varying factors; $I_{i, t}$ represents one of the life insurance variables used above for country $i$ in year $t, C_{i t}$ stands for an array of economic determinants; $D_{i t}$ refers to an demographic determinants; $Q_{i t}$ is the institutional quality measure implemented. $\epsilon_{i t}$ is the normally distributed error term; $i$ and $t$ represent indexes for cross-sections and time series, respectively, whereby $i=1, \ldots, \mathrm{N}$ and $t=$ $1, \ldots, \mathrm{T}$.

This proposed model can be applied to test for all of three institutional quality categories under study.

In this study, for economic determinants, we use GNI per capita as an indicator of income level, real interest rate, inflation rate, banking sector development measured by private credits by banks as well as gross domestic savings as percentage of GDP as an indicator of wealth. Socialdemographic variables included are life expectancy, age dependency ratio in addition to tertiary schooling as a proxy for risk aversion. For political institutions, we use political stability and no violence index issued by World Governance Indicators (WGI) (Kaufmann \& Kraay, 2016), government integrity index by Heritage Foundation, government effectiveness index as well as political stability and absence of violence by International Country Risk Guide (Political Risk Services, 2016) (ICRG). For legal institutions, we use property rights index by Heritage Foundation (Heritage Foundation, 2016), law and order by ICRG and WGI's regulatory quality index. As for economic institutions we use business freedom index, financial freedom index and trade freedom which are all produced by Heritage Foundation.

For life insurance consumption, we use insurance penetration rate which is calculated by dividing life insurance premiums by real GDP. Using insurance penetration rate is omnipresent in life insurance related literature (Alhassan \& Biekpe, 2016; Beck \& Webb, 2003; Kjosevski, 2012; Sen \& Madheswaran, 2013). We also use insurance density rate which is calculated by dividing life insurance premiums by population. The usage of insurance density rate is frequent in life insurance (Beck \& Webb, 2003; Beenstock et al., 1986; Browne \& Kim, 1993; Elango \& Jones, 2011; Kjosevski, 2012; Sen \& Madheswaran, 2013; Ward \& Zurbruegg, 2002). 
The data sample used in this study consists of 33 OIC country. The selection of countries is based solely on data availability. The available data covers the span of 27 years between 1990 till 2016. This period is selected based on the availability of insurance and institutional data. Global Financial Development Indicators' database includes insurance data starting from the year 1990 only. Data for Heritage Foundation indexes and WGI are also limited. Data for the first covers the period between 1995 till 2016 while it is from 1996 till 2015 for the later. Lastly, whenever institutional variables from ICRG are used, they cover the years between 1990 till 2012. We follow Beck \& Webb (2003) who resort to using natural log transformation to allow for the coefficients to be interpreted as elasticities. Outliers has also been winsorised at $1 \%$ and $99 \%$ percentiles level by county.

\subsection{Empirical Model}

This research employs panel data approach. Panel data can handle inter-unit differences as well as intra-unit dynamics since the dataset contains both cross-sectional and time-series dimensions. According to Baltagi (2005, p. 4-7) and Hsiao (2005), panel data has various advantages over time-series and cross-sectional data such as controlling for unit's heterogeneity and improving efficiency of economic estimates by increasing the degrees of freedom while mitigating collinearity. The classic linear regression produces consistent point estimates of $\beta$ number of parameters when the regression model is denoted as follows:

$$
y_{i}=\beta_{0}+\beta_{1} x_{1 i}+\cdots+\beta_{k} x_{k i}+\epsilon_{i}, i=1,2,3, \ldots N
$$

Carries three assumptions: first, it assumes linearity of relationship between the dependent and independent variables, exogeneity of explanatory variables: (zero conditional mean): $\mathrm{E}\left(\epsilon_{i} \mid x_{1 i}\right.$, $\left.x_{2 i}, \ldots, x_{k i}\right)=0$. and requires no autocorrelation. i.e. $\operatorname{Cov}\left(\epsilon_{i}, \epsilon_{j}\right)=0$ and that error terms are homoscedastic: i.e. $\operatorname{Var} \epsilon_{i}=\operatorname{Var} y_{i} \mid x_{1 i}, x_{2 i}, \ldots, x_{k i}=\delta^{2}$. A violation to the multicollinearity condition (zero-conditional-mean) would render ordinary least squares (OLS) estimates biased and/or inefficient.

In a regression with omitted time-invariant characteristics $(\mu)$ which are equal across crosssections through time t but differ across cross-sections units such as the following:

$$
y=\beta_{0}+\beta_{1} x_{1}+\cdots+\beta_{k} x_{k}+\mu+\epsilon
$$

$\mu+\epsilon$ form what is called the composite disturbance term $(\omega)$. The equation can be thus rewritten as follows:

$$
y=\beta_{0}+\beta_{1} x_{1}+\cdots+\beta_{k} x_{k}+\omega
$$

$$
\omega=\mu+\epsilon
$$

The composite disturbance term $(\omega)$ comprises of the unobserved heterogeneity component $\mu$ and the idiosyncratic error component $\epsilon$.

Explanatory variables that are correlated with the unobserved heterogeneity component $\mu$ would be correlated with the composite disturbance term $(\omega)$, thus, OLS parameter estimates would produce biased inconsistent results due to the violation of the zero-conditional-mean assumption. Pooled OLS assumes that there is no significant cross-section nor significant temporal effects among the panel. This assumption is hard to meet among macroeconomic indicators. 
Fixed effects or Random effects models are commonly used in order to mitigate the potential bias due to the unobserved heterogeneity. If the explanatory variables are uncorrelated with the unobserved heterogeneity effect $\mu_{i}$, then fixed effects estimator is consistent although inefficient. Meanwhile, random effects estimator would be considered consistent and efficient granted the same condition is met. Whereas, if the explanatory variables were found to be corelated with omitted heterogeneity $\mu_{i}$, then fixed effects estimator would be consistent whilst random effects is inconsistent (Baum, 2006).

As a result of the above discussion and following the majority approach of the literature e.g. (Beck \& Webb, 2003; Elango \& Jones, 2011; Esho et al., 2004; Kjosevski, 2012; S. C. Park \& Lemaire, 2012; Trinh et al., 2016), this paper employs fixed effects or random effects models to estimate equation (1).

In order to identify the appropriate model for the estimation, Hausman test is utilized. The null hypothesis of Hausman test assumes that $\mu_{i}$ is uncorrelated with the independent variables. Therefore, fixed effects model is appropriate if the null hypothesis is rejected, because only fixed effects model gives consistent coefficients (Beck \& Webb, 2003). In this study, fixed effects model is used when the null hypothesis is rejected at 5\% significance level and random effects model otherwise. Moreover, the assumption of homoskedasticity of error terms were there is heteroscedasticity, as well as having autocorrelated disturbances would produce consistent but inefficient estimates and the standard errors of these estimates will be biased (Baltagi, 2005, pp. 79-84). Therefore, in an attempt to mitigate possible issues of heteroscedasticity in errors and autocorrelation, this paper shall estimate robust standard errors. It is not unusual to depend on robust standard errors to ensure valid statistical inferences when one or more of the fundamental assumption is violated (Hoechle, 2007). This is achievable in Stata 14 using vce(robust) option (Hoechle, 2007).

\subsection{EMPIRICAL FINDINGS}

\subsection{Descriptive Statistics And Correlations}

Table 1 shows the descriptive statistics of all dependent and independent variables used in this study. It can be seen that there is a large variation in macroeconomic and institutional performances across OIC countries which suggests that the use of pooled OLS is not appropriate.

Table 1: Descriptive statistics for all variables used in the study

\begin{tabular}{lrrrrr}
\hline & count & min & max & mean & sd \\
\hline \hline Dependent Variables: & & & & & \\
$\quad$ Life Insurance Penetration & 346 & -8.47 & 1.81 & -2.3 & 1.71 \\
$\quad$ Life Insurance Density & 323 & -6.3 & 5.75 & 1.13 & 2.4 \\
Economic Determinants: & & & & & \\
$\quad$ GNI per capita & 323 & 5.09 & 11.08 & 7.69 & 1.39 \\
$\quad$ Real interest rate & 253 & -65.52 & 34.08 & 5.59 & 9.97 \\
$\quad$ Inflation, consumer prices (annual \%) & 378 & -0.11 & 10.67 & 0.15 & 0.78 \\
$\quad$ Private Credit & 408 & 0.33 & 4.86 & 2.84 & 0.93 \\
$\quad$ Gross Domestic Savings (\% of GDP) & 421 & -51.78 & 79.58 & 20.55 & 18.79 \\
Demographic Determinants: & & & & & \\
$\quad$ Life expectancy & 441 & 3.58 & 4.37 & 4.15 & 0.16 \\
$\quad$ Age Dependency & 441 & 2.82 & 4.78 & 4.21 & 0.35 \\
$\quad$ Tertiary School Enrolment & 349 & -2.36 & 4.51 & 2.28 & 1.2 \\
Institutional Determinants: & & & & & \\
Political Institutions: & & & & & \\
$\quad$ WGI-Political Stability and No Violence & 349 & -0.4 & 4.54 & 3.17 & 0.97 \\
Page | 102 & & & & &
\end{tabular}


Heritage-Government Integrity

WGI-Government Effectiveness

ICRG-Government Effectiveness

ICRG-Political Stability And Absence Of Violence

Legal Institutions:

Heritage-Property Rights

ICRG-Law and Order

WGI-Regulatory Quality

Economic Institutions:

Heritage-Business Freedom

Heritage-Investment Freedom

Heritage-Financial Freedom

Heritage-Monetary Freedom

Heritage-Trade Freedom
Heritage-Labour Freedom

$\begin{array}{rrrrr}364 & 2.3 & 4.5 & 3.24 & 0.57 \\ 350 & 0.65 & 4.51 & 3.33 & 0.77 \\ 268 & 3.22 & 4.47 & 3.77 & 0.35 \\ 294 & 3.61 & 4.54 & 4.24 & 0.18 \\ & & & & \\ 362 & 1.61 & 4.5 & 3.53 & 0.47 \\ 330 & 2.81 & 4.61 & 3.99 & 0.36 \\ 350 & -0.39 & 4.46 & 3.33 & 0.83 \\ & & & & \\ 364 & 3 & 4.61 & 4.05 & 0.24 \\ 357 & 1.61 & 4.32 & 3.75 & 0.42 \\ 362 & 2.3 & 4.46 & 3.64 & 0.5 \\ 359 & 2.97 & 4.49 & 4.26 & 0.21 \\ 195 & 3 & 4.55 & 4.04 & 0.3 \\ 362 & 2.53 & 4.47 & 4.08 & 0.3\end{array}$

Similarly, table $2 \mathrm{~A}$ and $2 \mathrm{~B}$ includes the correlation matrix between the variables of the study. We notice the existence of high correlations between explanatory variables which suggests the importance to control for country-specific effects that might drive multiple or all of said variables (Beck \& Webb, 2003). Tables 2 and 3 support the usage of fixed effects and random effects models in the study. 
Table 2A: Correlation table for all variables

\begin{tabular}{|c|c|c|c|c|c|c|c|c|c|c|c|c|}
\hline & $\begin{array}{c}\text { L. Ins } \\
\text { Penetration }\end{array}$ & L. Ins Density & GNI p.c & Interest & Inflation & $\begin{array}{l}\text { Private } \\
\text { credit }\end{array}$ & GDS $\%$ & Life Exp & $\begin{array}{c}\text { Age } \\
\text { Dependenc } \\
y\end{array}$ & Education & $\begin{array}{c}\text { WGI- } \\
\text { Political } \\
\text { Stability }\end{array}$ & $\begin{array}{l}\text { H-Gov } \\
\text { Integrity }\end{array}$ \\
\hline IL. Ins Penetration & 1 & & & & & & & & & & & \\
\hline L. Ins Density & $0.8078^{*}$ & 1 & & & & & & & & & & \\
\hline GNI p.c & $0.1848^{*}$ & $0.7206^{*}$ & 1 & & & & & & & & & \\
\hline Interest & -0.0471 & $-0.1809 *$ & -0.1101 & 1 & & & & & & & & \\
\hline Inflation & $-0.1298^{*}$ & $-0.1261 *$ & -0.0083 & $-0.5859 *$ & 1 & & & & & & & \\
\hline Private credit & $0.6146^{*}$ & $0.7616^{*}$ & $0.5668 *$ & -0.0718 & $-0.1535^{*}$ & 1 & & & & & & \\
\hline GDS $\%$ & 0.0609 & $0.4624^{*}$ & $0.7212^{*}$ & $-0.1103^{*}$ & $-0.0932 *$ & $0.3037 *$ & 1 & & & & & \\
\hline Life Exp & $0.2006^{*}$ & $0.6219 *$ & $0.7245^{*}$ & $-0.1286^{*}$ & 0.0046 & $0.6716^{*}$ & $0.4435^{*}$ & 1 & & & & \\
\hline Age Dependency & $-0.1789 *$ & $-0.5864 *$ & $-0.8066^{*}$ & 0.0846 & -0.0033 & $-0.5801 *$ & $-0.5663^{*}$ & $-0.7431 *$ & 1 & & & \\
\hline Education & $0.1555^{*}$ & $0.5167^{*}$ & $0.6375^{*}$ & $-0.1271 *$ & 0.0777 & $0.4604^{*}$ & $0.3377 *$ & $0.8033^{*}$ & $-0.6210^{*}$ & 1 & & \\
\hline WGI-Political Stability & $0.1401 *$ & $0.3545^{*}$ & $0.3681 *$ & $0.1230^{*}$ & $-0.3369 *$ & $0.3403^{*}$ & $0.2909 *$ & $0.2090^{*}$ & $-0.2922 *$ & 0.0195 & 1 & \\
\hline H-Gov Integrity & $0.3120^{*}$ & $0.5579 *$ & $0.5773^{*}$ & -0.0084 & $-0.2142 *$ & $0.5546^{*}$ & $0.3883^{*}$ & $0.4523^{*}$ & $-0.4612^{*}$ & $0.3376^{*}$ & $0.2939 *$ & 1 \\
\hline WGI-Gov Effectiveness & $0.4481 *$ & $0.6667 *$ & $0.5956 *$ & 0.0566 & $-0.1453 *$ & $0.6431 *$ & $0.2593^{*}$ & $0.5131 *$ & $-0.4760 *$ & $0.3430 *$ & $0.4784 *$ & $0.6133^{*}$ \\
\hline ICRG-Gov Effectiveness & $0.4392 *$ & $0.6116^{*}$ & $0.5520^{*}$ & -0.0006 & -0.04 & $0.5556^{*}$ & $0.2419 *$ & $0.4996^{*}$ & $-0.4922 *$ & $0.3646^{*}$ & $0.2988^{*}$ & $0.4440^{*}$ \\
\hline ICRG-Political Stability & -0.0275 & $0.2451^{*}$ & $0.3479 *$ & 0.0983 & $-0.3588^{*}$ & $0.2487 *$ & $0.2975^{*}$ & $0.2414^{*}$ & $-0.2844^{*}$ & 0.0432 & $0.8226^{*}$ & $0.2201 *$ \\
\hline H-Property Rights & $0.3389 *$ & $0.4571 *$ & $0.3657 *$ & 0.0066 & -0.0064 & $0.2895^{*}$ & 0.0475 & $0.1358 *$ & $-0.1268^{*}$ & 0.0066 & $0.2519 *$ & $0.4430^{*}$ \\
\hline ICRG-Law and Order & 0.061 & $0.4741^{*}$ & $0.5444^{*}$ & 0.0961 & $-0.1771 *$ & $0.4357^{*}$ & $0.3871 *$ & $0.4776^{*}$ & $-0.4555^{*}$ & $0.3631 *$ & $0.4814^{*}$ & $0.4154^{*}$ \\
\hline WGI-Regulatory Quality & $0.4070^{*}$ & $0.4961 *$ & $0.4114 *$ & $0.1419 *$ & $-0.2389 *$ & $0.4615^{*}$ & 0.0263 & $0.2007 *$ & $-0.2338^{*}$ & $0.1133^{*}$ & $0.3919 *$ & $0.5829 *$ \\
\hline H-Business Freedom & $0.1838^{*}$ & $0.4012^{*}$ & $0.4370^{*}$ & 0.0905 & 0.0563 & $0.3281 *$ & 0.0622 & $0.3255^{*}$ & $-0.3077 *$ & $0.3546^{*}$ & 0.0861 & $0.4036^{*}$ \\
\hline H-Investment Freedom & $0.2471 *$ & 0.0971 & -0.054 & $0.1671 *$ & -0.0442 & 0.0879 & $-0.2345^{*}$ & $-0.0909 *$ & $0.1233^{*}$ & $-0.1425^{*}$ & $0.1171 *$ & $0.2382 *$ \\
\hline H-Financial Freedom & $0.3004 *$ & $0.2344 *$ & $0.1648^{*}$ & $0.2351 *$ & -0.0588 & $0.2193^{*}$ & $-0.1470 *$ & -0.0023 & -0.0318 & -0.0288 & 0.0847 & $0.4301 *$ \\
\hline H-Monetary Freedom & $0.2446^{*}$ & $0.2146^{*}$ & $0.1211^{*}$ & $0.1462^{*}$ & $-0.7662^{*}$ & $0.3843^{*}$ & $0.1339 *$ & 0.0707 & -0.0793 & -0.0766 & $0.2606^{*}$ & $0.3426^{*}$ \\
\hline H-Labour Freedom & 0.019 & $0.2095^{*}$ & $0.3303^{*}$ & $0.1523^{*}$ & 0.0755 & $0.2323^{*}$ & 0.109 & $0.1921 *$ & $-0.2338 *$ & $0.2622^{*}$ & 0.0141 & $0.2916^{*}$ \\
\hline H-Trade Freedom & 0.0861 & $0.1897 *$ & $0.2823 *$ & -0.0227 & $-0.1067^{*}$ & $0.3293 *$ & $0.1439 *$ & $0.2874 *$ & $-0.3739 *$ & $0.2954 *$ & 0.0248 & $0.2826^{*}$ \\
\hline
\end{tabular}


Table 2B: Correlation Table For All Variables - OIC Countries

\begin{tabular}{|c|c|c|c|c|c|c|c|c|c|c|c|}
\hline & $\begin{array}{l}\text { WGI-Gov } \\
\text { Effectiveness }\end{array}$ & $\begin{array}{l}\text { ICRG-Gov } \\
\text { Effectiveness }\end{array}$ & $\begin{array}{l}\text { ICRG- } \\
\text { Political } \\
\text { Stability }\end{array}$ & $\begin{array}{l}\text { H-Property } \\
\text { Rights }\end{array}$ & $\begin{array}{l}\text { ICRG-Law } \\
\text { and Order }\end{array}$ & $\begin{array}{c}\text { WGI- } \\
\text { Regulatory } \\
\text { Quality }\end{array}$ & $\begin{array}{c}\text { H- } \\
\text { Business } \\
\text { Freedom }\end{array}$ & $\begin{array}{c}\mathrm{H}- \\
\text { Investment } \\
\text { Freedom }\end{array}$ & $\begin{array}{c}\text { H- } \\
\text { Financial } \\
\text { Freedom }\end{array}$ & $\begin{array}{l}\text { H-Monetary H-Labour } \\
\text { Freedom Freedom }\end{array}$ & $\begin{array}{l}\text { H-Trade } \\
\text { Freedom }\end{array}$ \\
\hline "WGI-Gov Effectiveness & 1 & & & & & & & & & & \\
\hline ICRG-Gov Effectiveness & $0.6503 *$ & 1 & & & & & & & & & \\
\hline ICRG-Political Stability & $0.4656^{*}$ & $0.2295^{*}$ & 1 & & & & & & & & \\
\hline H-Property Rights & $0.5687^{*}$ & $0.3210 *$ & $0.2981 *$ & 1 & & & & & & & \\
\hline ICRG-Law and Order & $0.5083^{*}$ & $0.3627 *$ & $0.5362 *$ & $0.2657 *$ & 1 & & & & & & \\
\hline WGI-Regulatory Quality & $0.7524^{*}$ & $0.4301 *$ & $0.4349 *$ & $0.7118^{*}$ & $0.3647 *$ & 1 & & & & & \\
\hline H-Business Freedom & $0.5528^{*}$ & $0.3585^{*}$ & $0.1623^{*}$ & $0.5685^{*}$ & $0.3478^{*}$ & $0.4930^{*}$ & 1 & & & & \\
\hline H-Investment Freedom & $0.3672 *$ & $0.1211 *$ & 0.059 & $0.5024 *$ & -0.0454 & $0.6547^{*}$ & $0.3292 *$ & 1 & & & \\
\hline H-Financial Freedom & $0.4423 *$ & $0.2423 *$ & 0.0806 & $0.5949 *$ & 0.0532 & $0.7762 *$ & $0.3419 *$ & $0.6379 *$ & 1 & & \\
\hline H-Monetary Freedom & $0.2557 *$ & $0.1197 *$ & $0.3010^{*}$ & $0.1438^{*}$ & $0.1648^{*}$ & $0.4092^{*}$ & 0.0338 & $0.1584^{*}$ & $0.3200^{*}$ & 1 & \\
\hline
\end{tabular}

* significant at 10 percent level. 


\subsection{Empirical Results}

The results of the empirical testing can be found in table 3 which uses life insurance penetration as dependent variable. Similarly, table 4 includes the results using life insurance density as robustness test. The results show that for economic determinants, income level measured by GNI per capita is not a significant determinant of life insurance consumption when measured by insurance penetration but is significant with positive impact when measured by insurance density. Considering the later, the results are consistent with general theoretical and empirical studies (Alhassan \& Biekpe, 2016; Beck \& Webb, 2003; Browne \& Kim, 1993; Elango \& Jones, 2011; Kjosevski, 2012; H. Park et al., 2002; Sen \& Madheswaran, 2013; Ward \& Zurbruegg, 2002). Similarly, real interest rate does not seem be a significant determinant of life insurance consumption in OIC countries even at 10\% significance level. This finding confirms suggested results of Kjosevski (2012) who found an inconclusive result regarding the impact of interest rate. That said, inflation rate also does not seem to significantly impact life insurance consumption in OIC countries which is against mainstream literature and theoretical suggestions. Additionally, banking sector development measured by private credit shows a positive impact as seen in models 3,4,5 and 12 in Table 3. This confirms other empirical studies such as Beck \& Webb (2003); Kjosevski (2012); Sen \& Madheswaran (2013). The results also suggest that there is a positive impact of gross domestic savings on life insurance consumption. This is consistent with findings of Beck \& Webb (2003) and Hammond et al. (1967) among others.

As for demographic determinants, life expectancy seems to follow the hypothesised sign which goes in line with findings of Alhassan \& Biekpe (2016); Beck \& Webb (2003); Sen \& Madheswaran (2013). Age dependency seems to have a positive impact on life insurance consumption as shown by Beck \& Webb (2003); Beenstock et al. (1986); Browne \& Kim (1993). Lastly, education shows a significant positive impact on life insurance consumption in congruent with findings of Beck \& Webb (2003); Hammond et al. (1967); Kjosevski (2012); Truett \& Truett (1990); Ward \& Zurbruegg (2002) 
Author / UMRAN - International Journal of Islamic and Civilizational Studies. vol. $\mathrm{x}$, no.x (2018) pp. xx - xx

Table 3: Fixed effects and random effects tests using life insurance penetration as dependent variable

\begin{tabular}{|c|c|c|c|c|c|c|c|c|c|c|c|c|c|}
\hline & (1) & (2) & (3) & (4) & (5) & (6) & (7) & (8) & (9) & (10) & (11) & $(12)$ & (13) \\
\hline \multirow[t]{2}{*}{ GNI p.c } & 1.229 & 0.581 & $1.309^{c}$ & 0.014 & 0.994 & 0.018 & 0.979 & $1.527^{\mathrm{c}}$ & 1.023 & 0.295 & 1.056 & -0.348 & 1.071 \\
\hline & [0.772] & [0.608] & {$[0.745]$} & {$[0.252]$} & {$[0.748]$} & {$[0.278]$} & {$[0.730]$} & [0.898] & [0.663] & [0.529] & [0.695] & [0.636] & [0.683] \\
\hline \multirow[t]{2}{*}{ Interest rate } & 0.011 & 0.027 & 0.005 & -0.005 & 0.025 & 0.007 & 0.011 & 0.022 & 0.027 & 0.025 & 0.021 & -0.018 & 0.024 \\
\hline & [0.014] & {$[0.017]$} & [0.015] & {$[0.012]$} & {$[0.018]$} & [0.019] & [0.015] & {$[0.017]$} & [0.018] & [0.017] & {$[0.017]$} & [0.014] & {$[0.017]$} \\
\hline \multirow[t]{2}{*}{ Inflation } & -3.98 & -0.143 & -2.554 & $-5.745^{b}$ & -0.899 & $-4.392^{b}$ & -3.87 & -0.64 & -2.018 & -0.711 & -1.259 & -4.03 & -1.204 \\
\hline & [2.735] & [2.122] & [2.695] & [2.294] & [2.345] & [1.944] & [2.774] & [2.188] & [2.383] & [2.161] & [2.118] & [2.823] & [2.209] \\
\hline \multirow[t]{2}{*}{ Private Credit } & 0.521 & 0.288 & $0.486^{\mathrm{b}}$ & $1.129^{\mathrm{a}}$ & 0.4 & $0.891^{\mathrm{a}}$ & 0.418 & 0.357 & 0.347 & 0.345 & 0.486 & $0.568^{c}$ & 0.385 \\
\hline & [0.463] & {$[0.472]$} & [0.223] & {$[0.224]$} & {$[0.487]$} & {$[0.275]$} & {$[0.478]$} & {$[0.464]$} & [0.468] & [0.411] & [0.358] & [0.310] & [0.487] \\
\hline \multirow[t]{2}{*}{ GDS $\%$} & $0.035^{\mathrm{c}}$ & $0.046^{\mathrm{b}}$ & -0.004 & 0.002 & $0.044^{b}$ & -0.004 & 0.027 & $0.043^{b}$ & $0.038^{c}$ & $0.053^{\mathrm{b}}$ & $0.046^{\mathrm{b}}$ & 0.003 & $0.043^{\mathrm{b}}$ \\
\hline & {$[0.020]$} & [0.019] & {$[0.015]$} & {$[0.013]$} & {$[0.020]$} & {$[0.014]$} & [0.019] & {$[0.020]$} & [0.019] & {$[0.021]$} & {$[0.022]$} & {$[0.017]$} & {$[0.020]$} \\
\hline \multirow[t]{2}{*}{ Life Exp } & -1.221 & -3.673 & -2.156 & $-2.238^{c}$ & -5.05 & -1.812 & -1.2 & $-6.129^{c}$ & -4.384 & $-4.657^{c}$ & $-5.393^{c}$ & 1.863 & -4.279 \\
\hline & [3.683] & [3.197] & [2.113] & [1.265] & {$[3.528]$} & [1.867] & [3.454] & [3.584] & [3.197] & [2.474] & [3.137] & {$[5.800]$} & [3.699] \\
\hline \multirow[t]{2}{*}{ Age Dependency } & 1.023 & $1.489^{c}$ & 0.23 & $1.272^{\mathrm{c}}$ & $1.971^{b}$ & 1.044 & 1.198 & $1.389^{c}$ & $2.303^{b}$ & $2.116^{\mathrm{b}}$ & $1.948^{\mathrm{b}}$ & 0.545 & $2.097^{b}$ \\
\hline & {$[0.754]$} & [0.808] & {$[0.786]$} & {$[0.686]$} & {$[0.826]$} & {$[0.773]$} & {$[0.857]$} & [0.769] & [0.892] & {$[0.855]$} & {$[0.824]$} & {$[0.710]$} & {$[0.855]$} \\
\hline \multirow[t]{2}{*}{ Education } & $0.799 \mathrm{c}$ & $0.712^{\mathrm{c}}$ & 0.26 & 0.352 & $0.772^{\mathrm{c}}$ & $0.436^{c}$ & $0.850^{c}$ & $0.783^{c}$ & $0.790^{c}$ & $0.787^{c}$ & $0.751^{\mathrm{c}}$ & $0.453^{c}$ & $0.750^{c}$ \\
\hline & {$[0.458]$} & [0.399] & {$[0.285]$} & {$[0.237]$} & [0.412] & {$[0.241]$} & {$[0.484]$} & {$[0.432]$} & {$[0.441]$} & [0.416] & [0.419] & {$[0.251]$} & [0.423] \\
\hline WGI-Political Stability & $\begin{array}{c}-0.273^{c} \\
{[0.145]}\end{array}$ & & & & & & & & & & & & \\
\hline H-Gov Integrity & & $\begin{array}{c}0.58 \\
{[0.365]}\end{array}$ & & & & & & & & & & & \\
\hline ICRG-Gov Effectiveness & & ] & $\begin{array}{c}1.660^{\mathrm{a}} \\
{[0.581]}\end{array}$ & & & & & & & & & & \\
\hline \multirow[t]{2}{*}{ ICRG-Political Stability } & & & & $-1.445^{b}$ & & & & & & & & & \\
\hline & & & & {$[0.576]$} & & & & & & & & & \\
\hline \multirow[t]{2}{*}{ H-Property Rights } & & & & & -0.065 & & & & & & & & \\
\hline & & & & & {$[0.425]$} & & & & & & & & \\
\hline \multirow[t]{2}{*}{ ICRG-Law and Order } & & & & & & -0.353 & & & & & & & \\
\hline & & & & & & {$[0.406]$} & & & & & & & \\
\hline \multirow[t]{2}{*}{ WGI-Regulatory Quality } & & & & & & & 0.283 & & & & & & \\
\hline & & & & & & & [0.198] & & & & & & \\
\hline H-Business Freedom & & & & & & & & $\begin{array}{l}-0.983 \\
{[0.645]}\end{array}$ & & & & & \\
\hline H-Investment Freedom & & & & & & & & & $\begin{array}{c}0.443^{c} \\
{[0.235]}\end{array}$ & & & & \\
\hline
\end{tabular}


H-Financial Freedom

H-Monetary Freedom

H-Labour Freedom

H-Trade Freedom

Constant

Obs

Countries

R2within

Hausman

Model used

IDT $=1$ means Non-OIC Developed Countries

c $p<0.1,{ }^{\mathrm{b}} p<0.05,{ }^{\mathrm{a}} p<0.01$

Table 4: Fixed effects and random effects tests using life insurance density as dependent variable (robustness tests)

\begin{tabular}{|c|c|c|c|c|c|c|c|c|c|c|c|c|c|}
\hline & (1) & (2) & (3) & (4) & (5) & (6) & $(7)$ & (8) & (9) & $(10)$ & $(11)$ & $(12)$ & (13) \\
\hline GNI p.c & $\begin{array}{l}2.084^{b} \\
{[0.795]}\end{array}$ & $\begin{array}{c}1.403^{\mathrm{b}} \\
{[0.628]}\end{array}$ & $\begin{array}{l}2.196^{\mathrm{a}} \\
{[0.770]}\end{array}$ & $\begin{array}{c}0.996^{a} \\
{[0.252]}\end{array}$ & $\begin{array}{c}1.828^{\mathrm{b}} \\
{[0.771]}\end{array}$ & $\begin{array}{c}0.988^{\mathrm{a}} \\
{[0.280]}\end{array}$ & $\begin{array}{c}1.821^{\mathrm{b}} \\
{[0.756]}\end{array}$ & $\begin{array}{l}2.389^{\mathrm{b}} \\
{[0.914]}\end{array}$ & $\begin{array}{c}1.853^{\mathrm{b}} \\
{[0.684]}\end{array}$ & $\begin{array}{l}1.096^{c} \\
{[0.544]}\end{array}$ & $\begin{array}{c}1.902^{\mathrm{b}} \\
{[0.713]}\end{array}$ & $\begin{array}{c}0 \\
{[0.636]}\end{array}$ & $\begin{array}{l}1.905^{\mathrm{b}} \\
{[0.707]}\end{array}$ \\
\hline Interest rate & $\begin{array}{c}0.011 \\
{[0.014]}\end{array}$ & $\begin{array}{c}0.027 \\
{[0.018]}\end{array}$ & $\begin{array}{c}0.005 \\
{[0.016]}\end{array}$ & $\begin{array}{c}-0.005 \\
{[0.012]}\end{array}$ & $\begin{array}{c}0.025 \\
{[0.018]}\end{array}$ & $\begin{array}{c}0.007 \\
{[0.019]}\end{array}$ & $\begin{array}{c}0.01 \\
{[0.015]}\end{array}$ & $\begin{array}{c}0.023 \\
{[0.018]}\end{array}$ & $\begin{array}{c}0.028 \\
{[0.018]}\end{array}$ & $\begin{array}{c}0.025 \\
{[0.017]}\end{array}$ & $\begin{array}{c}0.021 \\
{[0.017]}\end{array}$ & $\begin{array}{l}-0.018 \\
{[0.015]}\end{array}$ & $\begin{array}{c}0.025 \\
{[0.018]}\end{array}$ \\
\hline Inflation & $\begin{array}{l}-3.928 \\
{[2.726]}\end{array}$ & $\begin{array}{c}0.011 \\
{[2.122]}\end{array}$ & $\begin{array}{l}-2.554 \\
{[2.695]}\end{array}$ & $\begin{array}{l}-5.726^{\mathrm{b}} \\
{[2.243]}\end{array}$ & $\begin{array}{l}-0.782 \\
{[2.342]}\end{array}$ & $\begin{array}{l}-4.361^{\mathrm{b}} \\
{[1.931]}\end{array}$ & $\begin{array}{l}-3.811 \\
{[2.763]}\end{array}$ & $\begin{array}{l}-0.501 \\
{[2.182]}\end{array}$ & $\begin{array}{l}-1.857 \\
{[2.374]}\end{array}$ & $\begin{array}{l}-0.575 \\
{[2.150]}\end{array}$ & $\begin{array}{l}-1.202 \\
{[2.099]}\end{array}$ & $\begin{array}{c}-3.854 \\
{[2.791]}\end{array}$ & $\begin{array}{l}-1.058 \\
{[2.213]}\end{array}$ \\
\hline Private Credit & $\begin{array}{c}0.557 \\
{[0.474]}\end{array}$ & $\begin{array}{c}0.304 \\
{[0.482]}\end{array}$ & $\begin{array}{c}0.506^{\mathrm{b}} \\
{[0.224]}\end{array}$ & $\begin{array}{c}1.140^{\mathrm{a}} \\
{[0.228]}\end{array}$ & $\begin{array}{c}0.421 \\
{[0.498]}\end{array}$ & $\begin{array}{c}0.894^{\mathrm{a}} \\
{[0.283]}\end{array}$ & $\begin{array}{c}0.446 \\
{[0.490]}\end{array}$ & $\begin{array}{c}0.375 \\
{[0.474]}\end{array}$ & $\begin{array}{c}0.367 \\
{[0.479]}\end{array}$ & $\begin{array}{c}0.363 \\
{[0.418]}\end{array}$ & $\begin{array}{c}0.522 \\
{[0.366]}\end{array}$ & $\begin{array}{c}0.588^{c} \\
{[0.317]}\end{array}$ & $\begin{array}{c}0.406 \\
{[0.499]}\end{array}$ \\
\hline GDS $\%$ & $\begin{array}{c}0.038^{c} \\
{[0.021]}\end{array}$ & $\begin{array}{c}0.049^{\mathrm{b}} \\
{[0.020]}\end{array}$ & $\begin{array}{l}-0.004 \\
{[0.015]}\end{array}$ & $\begin{array}{c}0.003 \\
{[0.012]}\end{array}$ & $\begin{array}{c}0.047^{\mathrm{b}} \\
{[0.021]}\end{array}$ & $\begin{array}{c}-0.002 \\
{[0.014]}\end{array}$ & $\begin{array}{c}0.029 \\
{[0.020]}\end{array}$ & $\begin{array}{c}0.046^{\mathrm{b}} \\
{[0.020]}\end{array}$ & $\begin{array}{c}0.041^{\mathrm{c}} \\
{[0.020]}\end{array}$ & $\begin{array}{l}0.056^{\mathrm{b}} \\
{[0.022]}\end{array}$ & $\begin{array}{l}0.049^{\mathrm{b}} \\
{[0.023]}\end{array}$ & $\begin{array}{c}0.006 \\
{[0.018]}\end{array}$ & $\begin{array}{l}0.046^{\mathrm{b}} \\
{[0.021]}\end{array}$ \\
\hline Life expectancy & $\begin{array}{l}-1.284 \\
{[3.833]}\end{array}$ & $\begin{array}{l}-3.853 \\
{[3.338]}\end{array}$ & $\begin{array}{l}-2.177 \\
{[2.204]}\end{array}$ & $\begin{array}{l}-2.338^{c} \\
{[1.288]}\end{array}$ & $\begin{array}{l}-5.317 \\
{[3.698]}\end{array}$ & $\begin{array}{l}-1.869 \\
{[1.913]}\end{array}$ & $\begin{array}{l}-1.269 \\
{[3.589]}\end{array}$ & $\begin{array}{l}-6.412^{c} \\
{[3.720]}\end{array}$ & $\begin{array}{c}-4.601 \\
{[3.336]}\end{array}$ & $\begin{array}{l}-4.868^{c} \\
{[2.573]}\end{array}$ & $\begin{array}{l}-5.706^{c} \\
{[3.287]}\end{array}$ & $\begin{array}{c}1.367 \\
{[5.918]}\end{array}$ & $\begin{array}{c}-4.53 \\
{[3.856]}\end{array}$ \\
\hline Age Dependency & $\begin{array}{c}1.082 \\
{[0.777]}\end{array}$ & $\begin{array}{l}1.565^{\mathrm{c}} \\
{[0.817]}\end{array}$ & $\begin{array}{c}0.246 \\
{[0.803]}\end{array}$ & $\begin{array}{l}1.255^{\mathrm{c}} \\
{[0.681]}\end{array}$ & $\begin{array}{l}2.065^{\mathrm{b}} \\
{[0.849]}\end{array}$ & $\begin{array}{c}1.037 \\
{[0.762]}\end{array}$ & $\begin{array}{c}1.268 \\
{[0.887]}\end{array}$ & $\begin{array}{c}1.453^{\mathrm{c}} \\
{[0.785]}\end{array}$ & $\begin{array}{l}2.403^{b} \\
{[0.918]}\end{array}$ & $\begin{array}{l}2.216^{\mathrm{b}} \\
{[0.882]}\end{array}$ & $\begin{array}{l}2.038^{\mathrm{b}} \\
{[0.839]}\end{array}$ & $\begin{array}{c}0.63 \\
{[0.717]}\end{array}$ & $\begin{array}{l}2.183^{b} \\
{[0.878]}\end{array}$ \\
\hline Education & $\begin{array}{c}0.803^{\mathrm{c}} \\
{[0.467]}\end{array}$ & $\begin{array}{c}0.724^{c} \\
{[0.405]}\end{array}$ & $\begin{array}{c}0.247 \\
{[0.290]}\end{array}$ & $\begin{array}{c}0.359 \\
{[0.234]}\end{array}$ & $\begin{array}{c}0.790^{c} \\
{[0.420]}\end{array}$ & $\begin{array}{c}0.459^{c} \\
{[0.237]}\end{array}$ & $\begin{array}{c}0.857^{c} \\
{[0.494]}\end{array}$ & $\begin{array}{c}0.799 c \\
{[0.440]}\end{array}$ & $\begin{array}{c}0.813^{\mathrm{c}} \\
{[0.449]}\end{array}$ & $\begin{array}{c}0.803^{\mathrm{c}} \\
{[0.422]}\end{array}$ & $\begin{array}{c}0.764^{c} \\
{[0.424]}\end{array}$ & $\begin{array}{c}0.458^{c} \\
{[0.253]}\end{array}$ & $\begin{array}{l}0.765^{c} \\
{[0.431]}\end{array}$ \\
\hline WGI-Political Stability & $-0.292^{c}$ & & & & & & & & & & & & \\
\hline
\end{tabular}

Page 108
0.928

$$
\text { [0.556] }
$$

$-0.852$

$-0.209$

[0.284]

$-0.566 c$

$[0.308$

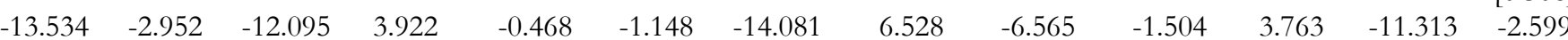

$\left[\begin{array}{lllllllllllll}15.586] & {[12.943]} & {[7.708]} & {[5.998]} & {[16.642]} & {[7.245]} & {[14.708]} & {[13.631]} & {[12.016]} & {[11.047]} & {[13.322]} & {[21.374]} & {[14.243}\end{array}\right.$

$\begin{array}{ccccccccccccc}124 & 123 & 107 & 113 & 123 & 104 & 124 & 123 & 121 & 123 & 123 & 90 & 123 \\ 33 & 33 & 27 & 29 & 33 & 29 & 33 & 33 & 33 & 33 & 33 & 31 & 33 \\ 0.493 & 0.513 & 0.663 & 0.595 & 0.489 & 0.539 & 0.48 & 0.507 & 0.505 & 0.533 & 0.492 & 0.351 & 0.496 \\ 0.001 & 0.001 & 0.019 & 0.071 & 0.002 & 0.152 & 0.003 & 0.001 & 0.001 & 0.001 & 0.002 & 0.036 & 0.001 \\ \text { FE } & \text { FE } & \text { FE } & \text { RE } & \text { FE } & \text { RE } & \text { FE } & \text { FE } & \text { FE } & \text { FE } & \text { FE } & \text { FE } & \text { FE }\end{array}$

FE

FE 6 
H-Government Integrity

ICRG-Government Effectivenes

ICRG-Political Stability

H-Property Rights

ICRG-Law and Order

WGI-Regulatory Quality

H-Business Freedom

H-Investment Freedom

H-Financial Freedom

H-Monetary Freedom

H-Labour Freedom

H-Trade Freedom

Constant

Obs

Countries

R2within

R2between

R2overall

Hausman

Model used

IDT=1 means Non-OIC Developed Countries c $p<0.1,{ }^{\mathrm{b}} p<0.05$, a $p<0.01$

[0.148]

$$
\begin{gathered}
0.6 \\
{[0.377]}
\end{gathered}
$$

$[0.582]$

$-1.501^{a}$

[0.581]

$$
-0.376
$$

[0.404]

0.297
$[0.201]$

$$
-1.031
$$$$
\text { [0.651] }
$$

$0.451^{\mathrm{c}}$

[0.241]

$0.975^{c}$

$-1.003$

[1.740]

$-0.145$

[0.284]

$-0.538$

$\begin{array}{ccccccccccccc}-17.107 & -6.013 & -16.008 c & 0.122 & -3.212 & -5.216 & -17.666 & 3.908 & -9.637 & -4.515 & 1.683 & -12.973 & -5.554 \\ {[16.128]} & {[13.450]} & {[7.933]} & {[6.222]} & {[17.286]} & {[7.402]} & {[15.186]} & {[14.075]} & {[12.504]} & {[11.413]} & {[13.898]} & {[21.967]} & {[14.799]}\end{array}$

$\begin{array}{llllllllllll}124 & 123 & 107 & 113 & 123 & 104 & 124 & 123 & 121 & 123 & 123 & 90\end{array}$

$\begin{array}{llllllllllll}33 & 33 & 27 & 29 & 33 & 29 & 33 & 33 & 33 & 33 & 33 & 31\end{array}$

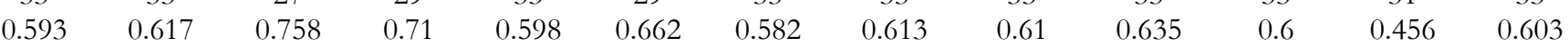

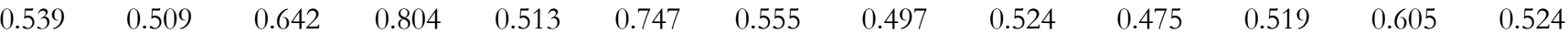

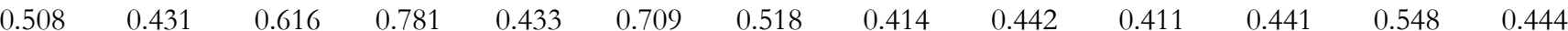

$\begin{array}{lllllllllllll}0.002 & 0.001 & 0.028 & 0.099 & 0.002 & 0.183 & 0.004 & 0.001 & 0.002 & 0.001 & 0.003 & 0.031 & 0.002\end{array}$

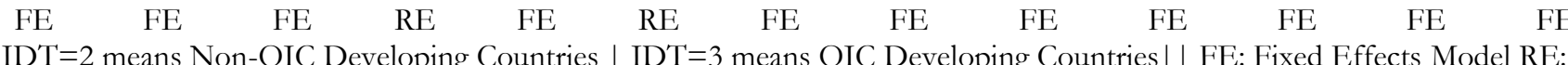

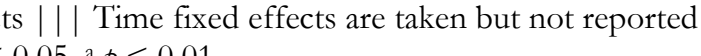




\subsubsection{Political Institutions}

As shown in the result of the main analysis in table 3 and the robustness tests in table 4 , the impact of political stability and absence of violence index by WGI and the corresponding index by ICRG appear to negatively impact life insurance consumption. Since higher score in these two indexes indicate a more stable country, it seems instability promotes life insurance consumption. Apparently, the lack of feeling of security might motivate individuals to pursue life insurance/family takaful coverage to avoid potential hardship for their families. Further, government effectiveness index issued by ICRG has positive impact on consumption of life insurance in OIC countries at 99 percent significance level. The result suggests that a 10 percent increase in government effectiveness would increase life insurance penetration by 16 percent and insurance density by 17 percent. The robustness tables confirm the finding of the main analysis.

\subsubsection{Legal Institutions}

Legal institutions do not seem to significantly affect life insurance consumption in OIC countries. none of the three indexes used to measure legal institutions suggested that their coefficients are significantly different than zero neither in the main analysis nor in the robustness test using life insurance density as dependent variable. The authors conducted further investigation using three other legal institutional indexes, namely: ICRG's voice and accountability index, WGI's rule of law index and regulatory quality index by ICRG. None of which suggested the impact of legal institutions to be significant. Results of the three extra variables mentioned are not reported.

\subsubsection{Economic Institutions}

The result suggests that investment freedom index has a robust positive impact on life insurance consumption in OIC countries at 90 percent significance level. Additionally, financial freedom index positively impacts life insurance density but not life insurance penetration. Finally, trade freedom index has a negative impact on life insurance consumption in OIC countries. This finding is against the hypothesis that more trade freedom might drive insurance consumption upwards. A possible explanation is that more trade freedom might allow individuals to increase their wealth leading for them to opt for being self-insured. Still, further investigation might be needed to explore the validity of this explanation. None of business freedom index, financial freedom index or monetary freedom index indicate any significant impact on life insurance consumption in OIC countries.

\subsection{CONCLUSION AND POLICY IMPLICATIONS}

This paper aims to test the impact of political, legal and economic institutions on life insurance consumption measured by life insurance penetration and life insurance density in OIC countries for the period between 1990 and 2016.

The results for political institutions suggest that in OIC countries, the more instable the country is, the more the consumption of life insurance/ family takaful is. Additionally, consumption for life insurance/ family takaful increases with the increase in government effectiveness. That said, the empirical finding does not support that legal institutions affect life insurance consumption. Lastly, the significance and direction of the impact of economic institutions on life insurance consumption is quite subjective to the proxy used. Life insurance 
consumption in OIC countries is positively affected by investment and financial freedom yet negatively impacted by trade freedom.

The finding of this paper serve to confirm the suggestion of Kunčič (2014) that the different aspects of institutions shall be considered rather than studying the impact of a comprehensive institutional quality index. The focus of policy makers in promoting life insurance shall not be limited to economic growth and other financial services but rather extended to cover both formal and informal institutions. Even though some of the findings suggest a negative impact of better institutions on life insurance consumption, better institutional quality would improve the overall economy by reducing uncertainty and therefore lowering risks as suggested by North (1990). This in turn will affect the economy on the macro and micro level which would eventually improve life insurance/ family takaful consumption.

Policy makers can focus on improving institutional quality in order to promote life insurance/ family takaful consumption. One plausible way is passing legislations allowing family takaful business for those countries that still do not offer such services and improving the regulatory framework for countries that already offer family takaful. The introduction and improvement of family takaful regulations might positively affect informal institutions towards more acceptance of life insurance. This is because it might help in reducing resentment fuelled by religious sentiment against life insurance services and toward better protection for the society.

\section{List of Reference}

Alhassan, A. L., \& Biekpe, N. (2016). Determinants of life insurance consumption in Africa. Research in International Business and Finance, 37, 17-27.

Anderson, J. E. (2005). Trade and Informal Institutions. Handbook Of International Trade, 2, 279-293.

Anderson, J. E., \& Marcouiller, D. (2002). Insecurity and the Pattern of Trade: An Empirical Investigation. Review of Economics and Statistics, 84(2), 342-352.

Baltagi, B. H. (2005). Econometric Analysis Of Panel Data. John Wiley \& Sons Ltd, Chichester.

Baum, C. F. (2006). An Introduction to Modern Econometrics Using Stata. STATA Press, 341. Retrieved from http://www.stata-press.com/books/modern-econometrics-stata/

Beck, T., \& Webb, I. (2003). Economic, demographic, and institutional determinants of life insurance consumption across countries. The World Bank Economic Review, 17(October), 51-88.

Beenstock, M., Dickinson, G., \& Khajuria, S. (1986). The Determination of Life Premiums: An International Cross Section Analysis. Insurance: Mathematics and Economics, 5, 261-270.

Beenstock, M., Dickinson, G., \& Khajuria, S. (1988). The Relationship Between Property-Liability Insurance Premiums and Income: An International Analysis. The Journal of Risk Insurance, 55(2), 259272.

Bhattacharyya, S., \& Hodler, R. (2014). Do Natural Resource Revenues Hinder Financial Development? The Role of Political Institutions. World Development, 57, 101-113.

Browne, M. J., Chung, J., \& Frees, E. W. (2000). International Property-Liability Insurance Consumption. The Journal of Risk and Insurance, 67(1), 73-90.

Browne, M. J., \& Kim, K. (1993). An International Analysis of Life Insurance Demand. The Journal of Risk and Insurance, 60(4), 616-634.

Brunetti, A., Kisunko, G., \& Weder, B. (1997). Institutions in Transition: Reliability of Rules and Economic Performance in Former Socialist Countries. Policy Research Working Paper Series 1809, The World Bank. 
Butkiewicz, J. L., \& Yanikkaya, H. (2006). Institutional quality and economic growth: Maintenance of the Rule Of Law Or Democratic Institutions, Or Both? Economic Modelling, 23(4), 648-661.

Chang, C.-H., \& Lee, C.-C. (2011). Non-Linearity Between Life Insurance and Economic Development: A Revisited Approach. The Geneva Risk and Insurance Review, 37(2), 223-257.

Chong, A., \& Calderón, C. (2000). Causality and Feedback between Institutional Measures and Economic Growth. Economics and Politics, 12(1), 69-81.

Crawford, S., Russignan, L., \& Kumar, K. (2018). Global Insurance Trends Analysis 2018 Ernst \& Young. June 2018 . [pdf]. Available at: https://www.ey.com/Publication/vwLUAssets/ey-global-insurancetrends-analysis-2018/\$File/ey-global-insurance-trends-analysis-2018.pdf . Assessed on 8 September 2019

Demetriades, P., \& Law, S. H. (2006). Finance, Institutions And Economic Development. International Journal of Finance and Economics, 11(3), 245-260.

Dollar, D., \& Kraay, A. (2003). Institutions, Trade, And Growth, Journal of Monetary Economics, 50, 133-162.

Elango, B., \& Jones, J. (2011). Drivers of Insurance Demand in Emerging Markets. Journal of Service Science Research, 3(December), 185-204.

Esho, N., Kirievsky, A., Ward, D., \& Zurbruegg, R. (2004). Law and the Determinants of Property-Casualty Insurance. Journal of Risk and Insurance, 71(2), 265-283.

Fischer, S. (1973). A Life Cycle Model of Life Insurance Purchases. International Economic Review, 14(1), 18 31.

Grigorian, D. a, \& Martinez, A. (2001). Industrial Growth and the Quality of Institutions: What do (transition) economies have to gain from the rule of law? Private and Financial Sector Development Unit Erope and Central Asia Region-World Bank, 5, 73.

Hakansson, N. H. (1969). Optimal Investment and Consumption Strategies Under Risk, an Uncertain Lifetime, and Insurance. International Economic Review, 10(3), 443-466.

Hammond, J. D., Houston, D. B., \& Melander, E. R. (1967). Determinants of Household Life Insurance Premium Expenditures: An Empirical Investigation. Journal of Risk and Insurance, 34(3), 397-408. https://doi.org/doi:10.2307/250854.

Heritage Foundation. (2016). The Index of Economic Freedom. Retrieved July 1, 2018, from https://www.heritage.org/index_/book/methodology

Hoechle, D. (2007). Robust Standard Errors For Panel Regressions With Cross-Sectional Dependence. Stata Journal, 7(3), 281-312.

Hsiao, C. (2005). Why Panel Data? The Singapore Economic Review, 50(02), 143-154.

Huber, P. J. (1967). The behavior of maximum likelihood estimates under nonstandard conditions. In Proceedings Of The Fifth Berkeley Symposium On Mathematical Statistics And Probability, 1, 221-233.

Kaufmann, D., \& Kraay, A. (2016). Worldwide Governance Indicators (www.govindicators.org). Retrieved July 1, 2018, from www.govindicators.org

Kjosevski, J. (2012). The Determinants of Life Insurance Demand in Central and Southeastern Europe. International Journal of Economics and Finance, 4(3), 237-247.

Klein, M. W., Alfaro, I. L., Block, S., Boggess, M., Goldberg, L., Marsh, L., \& Olivei, G. (2005). Capital Account Liberalization, Institutional Quality and Economic Growth: Theory and Evidence. Foreign Affairs. Retrieved from https://www.nber.org/papers/w11112

Knack, S., \& Keefer, P. (1995). Institutions and Economic Performance: Cross-Country Tests using Alternative Institutional Measures. Economics \& Politics, 7(3), 207-227.

Kunčič, A. (2014). Institutional Quality Dataset. Journal of Institutional Economics, Forthcoming, 10(1), 135-161. https://doi.org/10.1017/S1744137413000192

Page $\mid 112$ 
Law, S. H., \& Azman-Saini, W. N. W. (2008). The Quality of Institutions and Financial Development. MAPRA PERPER. Munich Personal RePEc Archive, (12107). Retrieved from https://ideas.repec.org/p/pra/mprapa/12107.html\%0A Date access : November 15, 2017

Lewis, F. D. (1989). Dependents and the Demand for Life Insurance. American Economic Review, 79(3), 452 467. https:// doi.org/10.1257/jep.6.3.79

Mantis, G., \& Farmer, R. N. (1968). Demand for Life Insurance. American Risk and Insurance Association, 35(2), 247-256.

Méon, P. G., \& Sekkat, K. (2008). Institutional quality and trade: Which institutions? Which trade? Economic Inquiry, 46(2), 227-240.

Méon, P., \& Sekkat, K. (2002). Does the Quality of Institutions Limit the MENA's Integration in the World Economy? The World Economy, 27(9), 1475-1498.

Mossin, J. (1968). Aspects of Rational Insurance Purchasing. Journal of Political Economy, 76(4, Part 1), 553568.

North, D. C. (1990). Institutions, Institutional Change, and Economic Performance. Cambridge University Press, 5(1), 1-153.

Outreville, J. F. (1999). Financial Development, Human Capital and Political Stability, (142). United Nations Conference on Trade and Development.

Park, H., Borde, S. F., \& Choi, Y. (2002). Determinants of insurance Pervasiveness: A Cross-National Analysis. International Business Review, 11(1), 79-96.

Park, S. C., \& Lemaire, J. (2012). The Impact of Culture on the Demand for Non-Life Insurance. Astin Bulletin, 42(2), 501-527.

Political Risk Services. (2016). International Country Risk Guide (ICRG). Retrieved July 1, 2018, from https://www.prsgroup.com/wp-content/uploads/2018/01/icrgmethodology.pdf

Pratt, J. W. (1964). Risk Aversion in the Small and in the Large. Econometrica, 32(1), 122-136.

Redek, T., \& Sušjan, A. (2005). The Impact of Institutions on Economic Growth: The Case of Transition Economies. Journal of Economic Issues, 39(4), 995-1027.

Rodrik, D. (2000). Institutions for high-quality growth: What They Are And How To Acquire Them. Studies in Comparative International Development, 35(3), 3-31.

Roe, M. J., \& Siegel, J. I. (2011). Political instability: Effects on financial development, roots in the severity of economic inequality. Journal of Comparative Economics, 39(3), 279-309.

Sen, S., \& Madheswaran, S. (2013). Regional determinants of life insurance consumption: Evidence from selected Asian economies. Asian-Pacific Economic Literature, 27(2), 86-103.

Siddiqui, D. A., \& Ahmed, Q. M. (2013). The effect of institutions on economic growth: A global analysis based on GMM dynamic panel estimation. Structural Change and Economic Dynamics, 24(1), 18-33. https://doi.org/10.1016/j.strueco.2012.12.001

Smith, V. L. (1968). Optimal Insurance Coverage`. Journal of Political Economy, 76(1), 68-77.

Trinh, T., Nguyen, X., \& Sgro, P. (2016). Determinants of Non-Life Insurance Expenditure In Developed And Developing Countries: An Empirical Investigation. Applied Econmics, 48(58), 5639-5653.

Truett, D. B., \& Truett, L. J. (1990). The demand for life insurance in Mexico and the United States: A comparative study. The Journal of Risk and Insurance, 57(2), 321-328. https:// doi.org/10.2307/253306

Ward, D., \& Zurbruegg, R. (2002). Law, Politics and Life Insurance Consumption in Asia. The Geneva Papers on Risk and Insurance, 27(3), 395-412.

White, H. (1980). A heteroskedasticity-consistent covariance matrix estimator and a direct test for heteroskedasticity. Econometrica: Journal of the Econometric Society, 817-838. 
Mohammad Ghaith Mahaini, Kamaruzaman Noordin \& Mohammad Taqiuddin Mohamad / UMRAN - International Journal of Islamic and Civilizational Studies. vol. 6, no.3 (2019) pp. 97- 114

Yaari, M. E. (1965). Uncertain Lifetime, Life Insurance, and the Theory of the Consumer. The Review of Economics Studies, 32(2), 137-150. 